\title{
Determinants of Diarrheal Disease among Adult People Living with HIV/AIDS Attending ART Clinics in Jimma Town, South-Western Ethiopia: A Case Control Study
}

\author{
Haimanot Ewnetu*, Wondwosen Kassahun and Desta Hiko \\ Department of Epidemiology, College of Public Health and Medical Sciences, Jimma University, Jimma, Ethiopia
}

*Corresponding author: Haimanot Ewnetu, Department of Epidemiology, College of Public Health and Medical Sciences, Jimma University, Jimma, Ethiopia, Tel: 251911983013; E-mail: hewnetu@yahoo.com

Received date: July 26, 2014; Accepted date: November 19, 2014; Published date: November 25, 2014

Copyright: (C) 2014 Ewnetu $\mathrm{H}$, et al. This is an open-access article distributed under the terms of the Creative Commons Attribution License, which permits unrestricted use, distribution, and reproduction in any medium, provided the original author and source are credited.

\section{Abstract}

Background: Human immunodeficiency virus/acquired immunodeficiency syndrome is among the leading causes of infectious diseases morbidity and mortality worldwide. A common manifestation of enteric involvement of HIV is diarrhea. Diarrhea affects approximately $90 \%$ of patients with AIDS in developing countries and $30-60 \%$ of AIDS patients in developed countries. The purpose of the study is to identify potential factors associated with occurrence of diarrheal diseases among adult peoples living with HIV who are currently attending ART clinics in Jimma town.

Methods: Institutional based unmatched case control study was conducted in Jimma town. A 95\% confidence interval is desired with $80 \%$ statistical power and $1: 1$ ratio of controls to cases. Samples were taken consecutively and data were collected using a interviewer-administered structured questionnaire. Bivariate analysis was done to identify candidates for multivariate analysis and multivariate analysis was done to identify potential risk factors of diarrheal diseases among adult peoples living with HIVIAIDS.

Results: Male sex (AOR=2.276; 95\% Cl:1.079, 4.800), public water point (AOR=4.972; 95\% Cl: 2.408, 10.266), absence of squat hole cover (AOR=2.763; 95\% Cl: 1.276, 5.980), waste disposal in garbage container (AOR=7.676; $95 \% \mathrm{Cl}: 1.776,33.188$ ), pets/animals in the house (AOR=2.260; $95 \% \mathrm{Cl}: 1.153,4.427$ ), not having refrigerator (AOR=3.343; 95\% Cl: $1.274,8.774)$, previous GIT disorder (AOR=4.254; 95\% Cl: 1.647, 10.987) and history of diarrhea (AOR=3.966; $95 \% \mathrm{Cl}: 1.896,8.295)$ were potential risk factors of diarrheal diseases.

Conclusions: Diarrheal disease were associated with gender, environmental and clinical factors such as public water point, non-use of latrine covers, presence of pets in the house, solid waste disposal in garbage container, absence of refrigerator, history of clinically diagnosed GIT disorder and previous history of diarrhea which suggested that hygiene and sanitation should be the core of basic preventive care package to prevent diarrheal disease.

Keywords: Determinants; Diarrheal diseases; HIV/AIDS

\section{Background}

Human immunodeficiency virus/acquired immunodeficiency syndrome is among the leading causes of infectious disease morbidity and mortality worldwide [1]. As a result, it is increasingly difficult to ignore the public health impact of human immunodeficiency virus/ acquired immunodeficiency syndrome. Even during current continuous advancement in Medicine; many still live with HIV. About 34 million people [31.6 million-35.2 million] were living with HIV worldwide by 2010. Sub-Saharan Africa region was the most affected area of the world by HIV/AIDS. Even though, the region contributes $12 \%$ of the global population, about $68 \%$ of all people living with HIV resided in this region in 2010. Moreover, 70\% of new HIV infections were recorded during 2010 in Sub-Saharan Africa [2]. Ethiopia is not an exception having HIV/AIDS prevalence of 1.5 among adults [3]. Ethiopia is not an exception, where the HIV/AIDS prevalence among adults is $1.5[3]$.
A common manifestation of enteric involvement of HIV is diarrhea leading to life threatening complications. Diarrhea can be defined as watery or liquid bowel movements that are more frequent than usual for an HIV/AIDS patient [4,5].

Diarrhea are occurring in highest rate in peoples living with HIV, 2-6 times higher than in those who are not infected, and rates of acute and persistent diarrhea are twice as high in infected than uninfected populations [6].

There is an increasing concern that the quality of life of people living with HIV is reduced due to infection which can also speed up the progression from HIV to AIDS. Moreover, diarrheal diseases challenge the progress of people living with HIV as it reduces the absorption of antiretroviral medicines and essential nutrients [7].

To date, it has been known that diarrheal diseases are endemic to areas with poor socio economic conditions (i.e. poor nutritional status, poor sanitation and lack of access to safe drinking water). Infectious diarrhea is the most prevalent illness in people infected with HIV [1]. In developed countries diarrhea occurs in $30-60 \%$ of AIDS patients and the figure is as high as $90 \%$ of AIDS patients in developing 
Page 2 of 9

countries. In a large proportion of this population, the diarrhea may become prolonged and life-threatening, and chronic diarrhea is an independent marker of poor prognosis in patients with AIDS $[1,5]$.

Chronic diarrhea (lasting for more than one month) being one of the major complaints of AIDS patients occurring in about $40 \%$ of cases, it is also one of the WHO-staging criteria for AIDS. It is not a life-threatening condition, but it can severely diminish quality of life. In conditions of poor sanitation it places a particularly heavy psychological and social burden on afflicted patients $[5,6]$.

As the AIDS pandemic has spread, diarrhea can cause significant morbidity in HIV-infected patients and can be due to a multitude of etiologies. There is limited information in Ethiopia on possible risk factors for diarrheal diseases in HIV-sero positive persons, although the importance of food and water safety in immune compromised populations is well known. The aim of this study was to address this knowledge gap by studying potential risk factors [7-9].

\section{Methods}

\section{Study setting and participants}

A case control study was conducted at Jimma University Specialized Hospital (JUSH) and Jimma Health Center which are located in Jimma town, $352 \mathrm{~km}$ southwest of Addis Ababa, the capital city, from January 20-March 23, 2013. The health institutions are the one which give ART services for Peoples Living with HIV/AIDS in Jimma town.

The source populations were all adult PLWHAs (Adult patients of 18 years or older) who have ever enrolled in the ART clinics including those who came for the first time during the study period. Cases were an HIV positive adult patient (18 years or older) presented with diarrhea whether or not on antiretroviral therapy and controls are an HIV positive adult (Adult patients of 18 years or older) patient without diarrhea at the time of presentation and without history of diarrhea in the preceding 14 days. The study populations for cases and controls were a sample of PLWHAs who were presented with and without diarrhea among those who attended the ART clinics during the study period, respectively. The reason the study focuses on adult peoples is that they account for large proportion of PLWHAs and are matured to handle the issues associated with HIV/AIDS and diarrheal diseases including the interventions and are cooperative.

In this study individuals who reported to pass more than three loose or watery stools within 24 hours period were considered to have diarrhea $[1,10]$.

\section{Sample size and sampling techniques}

The sample size was determined based on sample size calculation for two population proportions formula using Epi Info version 3.5.1.database and statistics software. Proportion of cases who had contact with pets and animals $(\mathrm{P} 1=87.1 \%)$ and the proportion of controls who had contact with pets and animals (P2 $=72.7 \%)$ [10] was taken and type I error rate (alpha) of 0.05 with $80 \%$ statistical power and 1:1 ratio of controls to cases. Finally a total of 268 samples (134 cases and 134 controls) were included in the study.

The total sample is allocated to the two health facilities according to the number of patients proportionally. Cases were taken consecutively while controls were selected using simple random sampling technique on daily basis from the ART clinic attendees and appointment registers using anonymous clinic attendance numbers assigned for the purposes of queuing.

\section{Data collection process}

Data were collected by trained nurses working in ART clinic using a structured and pre-tested questionnaire. The questionnaire was originally designed in English; and then translated to local languages and back translated to English by other person to check for its consistency.

After the study subjects were identified as cases and controls, they were sent to two separate rooms; one for cases and the other for controls but the data collectors are blinded for the status of the respondent. Then after, they were interviewed based on an interviewer administered structured questionnaire. Record review was used to collect information about some clinical factors such as: CD4 count, stage of the disease, ART regimen, opportunistic infections, history of poor adherence and cotrimoxazole prophylaxis.

The dependent variable was diarrheal diseases status among PLWHAs while the independent variables included socio demographic, water, hygiene and sanitation related and clinical variables.

\section{Data analysis}

Data were coded, then entered and cleaned in Epidata and analyzed using SPSS version 16 statistical software. Both descriptive and inferential statistical techniques were employed. Summary statistics such as percentages were computed using cross tabulation/ contingency table analysis and odds ratios were calculated with $95 \%$ confidence interval. Then bivariate analysis was done to test the association between the independent and the outcome variable. All explanatory variables that were associated with the outcome variable in bivariate analysis, at $\mathrm{P}<0.25$ were entered into multiple logistic regression model, based on backward LR technique using likelihood ratio test to identify the potential independent predictors of diarrhea among PLWHAs. $\mathrm{P}<0.05$ was considered as a cut-off point for statistical significance.

\section{Ethical consideration}

Ethical Clearance was obtained from Ethical Review Committee of Jimma University and verbal consent was taken from all the study participants.

\section{Results}

\section{Socio demographic characteristics of study subjects}

A total of 268 eligible subjects were enrolled, 134 cases (those with diarrhea) and 134 controls (those without diarrhea). Ninety (67.2\%) cases and $103(76.9 \%)$ controls were females. Ninety nine $(73.9 \%)$ cases and $111(82.8 \%)$ controls were in the age range of 25-45 years with the mean age of $35.5(\mathrm{SD}=9.3)$ and $33.7(\mathrm{SD}=7.9)$ respectively. Seventy seven $(57.5 \%)$ cases and 91 (67.9\%) controls were unemployed $(\mathrm{P}=0.069)$. Moreover, the majority of the study subjects, $91(67.9 \%)$ cases and $93(69.4 \%)$ controls earned 500ETB or less per month respectively.

One hundred one (75.4\%) cases and $110(82.1 \%)$ controls never chewed khat in the last one month $(\mathrm{P}=0.181)$. Similarly, more than 
Citation: Ewnetu H, Kassahun W, Hiko D (2014) Determinants of Diarrheal Disease among Adult People Living with HIVIAIDS Attending ART Clinics in Jimma Town, South-Western Ethiopia: A Case Control Study. J AIDS Clin Res 5: 380. doi:10.4172/2155-6113.1000380

Page 3 of 9

three fourth of 105 (78.4\%) cases and $114(85.1 \%)$ controls had no history of alcohol intake in the last one month. In addition, only 14 $(10.4 \%)$ cases and $15(11.2 \%)$ controls performed physical exercise at least twice per week (Table 1).

\section{Environmental risk factors}

Housing characteristics: The majority of respondents, 61 (45.5\%) cases and $68(50.7 \%)$ controls had a single room for the entire household members and almost all respondents, 120 (89.6\%) cases and 126 (94.0\%) controls had five or less usual household members. Accordingly, 33 (24.6\%) cases and 29 (21.6\%) controls live in crowded condition.

The study also revealed that $52(38.8 \%)$ cases and 70 (52.2\%) controls used charcoal and fire wood as source of energy for cooking (Table 2).

\begin{tabular}{|c|c|c|c|c|c|}
\hline Variable & Categories & $\begin{array}{l}\text { Cases } \\
\text { No (\%) }\end{array}$ & $\begin{array}{l}\text { Controls } \\
\text { No (\%) }\end{array}$ & $\begin{array}{l}\text { COR } \\
(95 \% \mathrm{Cl})\end{array}$ & P-value \\
\hline \multirow[t]{2}{*}{ Sex } & Female & $90(67.2)$ & $103(76.9)$ & 1.000 & \\
\hline & Male & $44(32.8)$ & $31(23.1)$ & $\begin{array}{l}1.624 \\
(0.94 \\
2.786)\end{array}$ & $0.078^{*}$ \\
\hline \multirow[t]{3}{*}{ Age } & $18-24$ & $11(8.2)$ & $10(7.5)$ & $\begin{array}{l}0.596 \\
(0.200,1 . \\
773)\end{array}$ & 0.352 \\
\hline & $25-45$ & 99 (73.9) & 111 (82.8) & $\begin{array}{l}0.483(0.2 \\
33,1.000)\end{array}$ & $0.050^{*}$ \\
\hline & $>45$ & $24(17.9)$ & $13(9.7)$ & 1.000 & \\
\hline \multirow[t]{2}{*}{ Residence } & Rural & $18(13.4)$ & $17(12.7)$ & $\begin{array}{l}1.068 \\
(0.525 \\
2.174)\end{array}$ & 0.856 \\
\hline & Urban & $116(86.6)$ & $117(87.3)$ & 1.000 & \\
\hline \multirow[t]{3}{*}{ Occupation } & Employed & $39(29.1)$ & $27(20.1)$ & 1.000 & \\
\hline & Merchant & $18(13.4)$ & 16 (11.9) & $\begin{array}{l}0.779 \\
(0.339 \\
1.792)\end{array}$ & 0.557 \\
\hline & Unemployed & $77(57.5)$ & $91(67.9)$ & $\begin{array}{l}0.586 \\
(0.329 \\
1.043)\end{array}$ & $0.069^{*}$ \\
\hline \multirow[t]{3}{*}{$\begin{array}{l}\text { Educational } \\
\text { status }\end{array}$} & $1-12$ & $51(38.1)$ & $68(50.7)$ & $\begin{array}{l}0.649 \\
(0.357 \\
1.178)\end{array}$ & $0.155^{*}$ \\
\hline & $\begin{array}{l}\text { Certificate } \\
\text { \&diploma }\end{array}$ & $46(34.3)$ & $34(25.4)$ & $\begin{array}{l}1.170 \\
(0.612 \\
2.238)\end{array}$ & 0.635 \\
\hline & Illiterate & $37(27.6)$ & 32 (23.9) & 1.000 & \\
\hline \multirow[t]{3}{*}{ Religion } & Orthodox & $71(53.0)$ & $64(47.8)$ & 1.000 & \\
\hline & Muslim & $38(28.4)$ & 37 (27.6) & $\begin{array}{l}1.464(0.7 \\
88, \\
2.722)\end{array}$ & 0.228 \\
\hline & Protestant & $25(18.7)$ & $33(24.6)$ & $\begin{array}{l}1.356(0.6 \\
81, \\
2.701)\end{array}$ & 0.387 \\
\hline
\end{tabular}

\begin{tabular}{|c|c|c|c|c|c|}
\hline \multirow[t]{4}{*}{ Ethnicity } & Oromo & 49 (36.6) & $54(40.3)$ & 1.000 & \\
\hline & Amhara & $32(23.9)$ & $28(20.9)$ & $\begin{array}{l}1.259 \\
(0.666 \\
2.383)\end{array}$ & 0.478 \\
\hline & Dawro & $29(21.6)$ & $28(20.9)$ & $\begin{array}{l}1.141 \\
(0.597 \\
2.181)\end{array}$ & 0.689 \\
\hline & Others ${ }^{*}$ & $24(17.9)$ & $24(17.9)$ & $\begin{array}{l}1.102 \\
(0.555 \\
2.187)\end{array}$ & 0.781 \\
\hline \multirow{4}{*}{$\begin{array}{l}\text { Marital } \\
\text { status }\end{array}$} & Single & $20(14.9)$ & $23(17.2)$ & 1.000 & \\
\hline & Married & 65 (48.5) & $58(43.3)$ & $\begin{array}{l}0.776 \\
(0.387 \\
1.556)\end{array}$ & 0.475 \\
\hline & Divorced & $22(16.4)$ & $29(21.6)$ & $\begin{array}{l}1.146 \\
(0.507 \\
2.593)\end{array}$ & 0.743 \\
\hline & Widowed & $27(20.1)$ & $24(17.9)$ & $\begin{array}{l}0.773 \\
(0.343 \\
1.743)\end{array}$ & 0.535 \\
\hline \multirow[t]{3}{*}{$\begin{array}{l}\text { Monthly } \\
\text { income }\end{array}$} & $\leq 500 \mathrm{ETB}$ & $91(67.9)$ & $93(69.4)$ & $\begin{array}{l}1.094 \\
(0.535 \\
2.236)\end{array}$ & 0.806 \\
\hline & 501-999ЕТВ & $26(19.4)$ & $22(16.4)$ & $\begin{array}{l}1.321 \\
(0.555 \\
3.141)\end{array}$ & 0.529 \\
\hline & $\geq 1000 \mathrm{ETB}$ & $17(12.7)$ & $19(14.2)$ & 1.000 & \\
\hline \multirow{2}{*}{$\begin{array}{l}\text { Khat } \\
\text { chewing in } \\
\text { the last } \\
\text { 1month }\end{array}$} & Yes & $33(24.6)$ & $24(17.9)$ & $\begin{array}{l}1.498 \\
(0.829 \\
2.705)\end{array}$ & $0.181^{*}$ \\
\hline & No & $101(75.4)$ & $110(82.1)$ & 1.000 & \\
\hline \multirow{3}{*}{$\begin{array}{l}\text { Alcohol } \\
\text { intake in the } \\
\text { last one } \\
\text { month }\end{array}$} & Once & $12(9.0)$ & $8(6.0)$ & $\begin{array}{l}1.629 \\
(0.64 \\
4.140)\end{array}$ & 0.306 \\
\hline & $\geq 2$ times/week & $17(12.7)$ & $12(9.0)$ & $\begin{array}{l}1.538 \\
(0.701 \\
3.372)\end{array}$ & 0.286 \\
\hline & Never & $105(78.4)$ & $114(85.1)$ & 1.000 & \\
\hline \multirow[t]{2}{*}{$\begin{array}{l}\text { Physical } \\
\text { exercise }\end{array}$} & Yes & $14(10.4)$ & $15(11.2)$ & $\begin{array}{l}0.926 \\
(0.428 \\
2.001)\end{array}$ & 0.844 \\
\hline & No & $120(89.6)$ & 119 (88.8) & 1.000 & \\
\hline $\begin{array}{l}\text { "Guraghe, Yem, } \\
\text { "Variables whic } \\
\mathrm{P}<0.25\end{array}$ & Kefa & 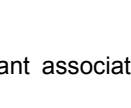 & . & & \\
\hline
\end{tabular}

Table 1: Univariate analysis for the association between sociodemographic characteristics and diarrheal diseases, Jimma town ART clinics, March 2013. 
Citation: Ewnetu H, Kassahun W, Hiko D (2014) Determinants of Diarrheal Disease among Adult People Living with HIVIAIDS Attending ART Clinics in Jimma Town, South-Western Ethiopia: A Case Control Study. J AIDS Clin Res 5: 380. doi:10.4172/2155-6113.1000380

Page 4 of 9

\section{Water supply and hygiene related factors}

The proportions of individuals who used protected water source was high among both cases $(82.8 \%)$ and controls $(92.5 \% \mathrm{P}=0.019)$, whereas cases $(65.7 \%)$ were more likely to use public water point compared with controls $(38.8 \%, \mathrm{P}<0.001)$. In other hands, $72(53.7 \%)$ cases and $90(67.2 \%)$ controls washed their hands before food preparation (Table 3 ).

\begin{tabular}{|c|c|c|c|c|c|}
\hline Variable & Categories & $\begin{array}{l}\text { Cases } \\
\text { No (\%) }\end{array}$ & $\begin{array}{l}\text { Controls } \\
\text { No (\%) }\end{array}$ & $\begin{array}{l}\text { COR } \\
(95 \% \mathrm{Cl})\end{array}$ & P-value \\
\hline \multirow[t]{2}{*}{ Roof } & Iron sheet & 125 (93.3) & $127(94.8)$ & 1.000 & \\
\hline & Grass & $9(6.7)$ & $7(5.2)$ & $\begin{array}{l}1.306 \\
(0.472, \\
3.616)\end{array}$ & 0.607 \\
\hline \multirow[t]{2}{*}{ Wall } & Cement/ Brick & $8(6.0)$ & $10(7.5)$ & 1.000 & \\
\hline & Mud/ Timber & $126(94.3)$ & $124(92.5)$ & $\begin{array}{ll}1.270 & 0(. \\
485, & \\
3.325) & \end{array}$ & 0.626 \\
\hline \multirow[t]{3}{*}{$\begin{array}{l}\text { No of } \\
\text { rooms }\end{array}$} & 1 & $61(45.5)$ & $68(50.7)$ & $\begin{array}{l}0.781 \\
(0.420 \\
1.454)\end{array}$ & 0.436 \\
\hline & 2 & $42(31.3)$ & $39(29.1)$ & $\begin{array}{l}0.938 \\
(0.477, \\
1.843)\end{array}$ & 0.853 \\
\hline & $\geq 3$ & $31(23.1)$ & 27 (20.1) & 1.000 & \\
\hline \multirow{2}{*}{$\begin{array}{l}\text { Usual } \\
\text { members } \\
\text { of the } \\
\text { househol } \\
\text { d }\end{array}$} & $\leq 5$ & $120(89.6)$ & $126(94.0)$ & 1.000 & \\
\hline & $>5$ & $14(10.4)$ & $8(6.0)$ & $\begin{array}{l}1.837 \\
(0.744, \\
4.537)\end{array}$ & $0.187^{*}$ \\
\hline \multirow{2}{*}{$\begin{array}{l}\text { Crowd } \\
\text { index }\end{array}$} & Uncrowded & $101(75.4)$ & $105(78.4)$ & 1.000 & \\
\hline & Over Crowded & $\begin{array}{l}33 \\
(24.6 \%)\end{array}$ & $29(21.6)$ & $\begin{array}{l}1.183 \\
(0.670, \\
2.089)\end{array}$ & 0.563 \\
\hline \multirow[t]{3}{*}{$\begin{array}{l}\text { Source of } \\
\text { energy }\end{array}$} & $\begin{array}{l}\text { Electricity/ } \\
\text { charcoal/ } \\
\text { kerosene }\end{array}$ & $54(40.3)$ & $48(35.8)$ & 1.000 & \\
\hline & $\begin{array}{l}\text { Charcoal/Fire } \\
\text { wood }\end{array}$ & $52(38.8)$ & $70(52.2)$ & $\begin{array}{l}0.670 \\
(0.394, \\
1.138)\end{array}$ & $0.138^{*}$ \\
\hline & Animal dung & $28(20.9)$ & $16(11.9)$ & $\begin{array}{l}1.464 \\
(0.715, \\
2.999)\end{array}$ & 0.297 \\
\hline $\begin{array}{l}\text { ariables } \\
<0.25 \text {. }\end{array}$ & & & & & Iysi \\
\hline
\end{tabular}

Table 2: Univariate analysis for the association between housing characteristics and diarrheal diseases, Jimma town ART clinics, March 2013.

\section{Sanitation related factors}

Almost all cases (89.6\%) and controls (88.8\%) had their own latrine and among those respondents who did not have their own latrine, 96.6\% use public /shared latrine and the rest (3.4\%) defecated on open field. Type of latrine $(\mathrm{P}=0.041)$, absence of latrine cover $(\mathrm{P}=0.027)$, solid waste disposal site $(\mathrm{P}=0.004)$, presence of pets in the house $(\mathrm{P}=0.104)$, contact with pets $(\mathrm{P}=0.233)$, presence of insects and rats at home $(\mathrm{P}=0.071)$, absence refrigerator in the house $(\mathrm{P}=0.006)$ and eating raw/uncooked food $(\mathrm{P}=0.015)$ had shown significant association during bivariate analysis (Table 4 ).

\begin{tabular}{|c|c|c|c|c|c|}
\hline Variable & Categories & $\begin{array}{l}\text { Cases } \\
\text { No (\%) }\end{array}$ & $\begin{array}{l}\text { Controls } \\
\text { No (\%) }\end{array}$ & $\begin{array}{l}\text { COR } \\
(95 \% \mathrm{Cl})\end{array}$ & P-value \\
\hline \multirow[t]{2}{*}{$\begin{array}{l}\text { Water } \\
\text { source }\end{array}$} & Protected & $\begin{array}{l}111 \\
(82.8)\end{array}$ & $124(92.5)$ & 1.000 & \\
\hline & Unprotected & $23(17.2)$ & $10(7.5)$ & $\begin{array}{l}2.569 \\
(1.172, \\
5.635)\end{array}$ & $0.019^{*}$ \\
\hline \multirow{2}{*}{$\begin{array}{l}\text { Water } \\
\text { point }\end{array}$} & Private & $46(34.3)$ & $82(61.2)$ & 1.000 & \\
\hline & Public & $88(65.7)$ & $52(38.8)$ & $\begin{array}{l}3.017(1 . \\
834 \\
4.963)\end{array}$ & $<0.001^{*}$ \\
\hline \multicolumn{6}{|c|}{ Water distance from house(in minutes) } \\
\hline & $<5$ minutes & $20(37.7)$ & $60(69.0)$ & 1.000 & \\
\hline & $>5$ minutes & $33(62.3)$ & $27(31.0)$ & $\begin{array}{l}3.667 \\
(1.789,7 . \\
514)\end{array}$ & $<0.001^{*}$ \\
\hline \multicolumn{6}{|c|}{ Daily water consumption(L) } \\
\hline & $>20 / p /$ day & $18(13.4)$ & $25(18.7)$ & 1.000 & \\
\hline & $<20 /$ p/day & $\begin{array}{l}116 \\
(86.6)\end{array}$ & $109(81.3)$ & $\begin{array}{l}1.234 \\
(0.65, \\
2.334)\end{array}$ & 0.517 \\
\hline \multicolumn{6}{|c|}{ Water storage container } \\
\hline & Jerikan & $\begin{array}{l}104 \\
(77.6)\end{array}$ & $118(88.1)$ & 1.000 & \\
\hline & Pot & $13(9.7)$ & $4(3.0)$ & $\begin{array}{l}3.687 \\
(1.166, \\
11.66)\end{array}$ & 0.026 * \\
\hline & Bucket/tanker & $17(12.7)$ & $12(9.0)$ & $\begin{array}{l}1.607 \\
(0.733, \\
3.523)\end{array}$ & 0.236 \\
\hline \multicolumn{6}{|c|}{ Treat non potable water } \\
\hline & Yes & $45(33.6)$ & $63(47.0)$ & 1.000 & \\
\hline & No & $89(66.4)$ & $71(53.0)$ & $\begin{array}{l}1.755 \\
(1.071, \\
2.875)\end{array}$ & $0.026^{*}$ \\
\hline \multicolumn{6}{|c|}{ Distance of water from latrine } \\
\hline & $>30$ meter & $36(26.9)$ & $25(18.7)$ & 1.000 & \\
\hline & $<30$ meter & $98(73.1)$ & $109(81.3)$ & $\begin{array}{l}1.602 \\
(0.898 \\
2.857)\end{array}$ & $0.111^{*}$ \\
\hline
\end{tabular}


Citation: Ewnetu H, Kassahun W, Hiko D (2014) Determinants of Diarrheal Disease among Adult People Living with HIVIAIDS Attending ART Clinics in Jimma Town, South-Western Ethiopia: A Case Control Study. J AIDS Clin Res 5: 380. doi:10.4172/2155-6113.1000380

Page 5 of 9

\begin{tabular}{|l|l|l|l|l|l|}
\hline \multicolumn{6}{|l}{ Hand washing before food preparation } \\
\hline & Yes & $72(53.7)$ & $90(67.2)$ & 1.000 & \\
\hline & No & $62(46.3)$ & $44(32.8)$ & 1.761 & $0.025^{*}$ \\
& & & & 2.073, & \\
& & & & $2.890)$ & \\
\hline
\end{tabular}

Facility used for hand washing

\begin{tabular}{|l|l|l|l|l|l|} 
& Soap & $\begin{array}{l}103 \\
(76.9)\end{array}$ & $122(91.0)$ & 1.000 & \\
\hline & Ash & $11(8.2)$ & $6(4.5)$ & $\begin{array}{l}2.172 \\
(0.776, \\
6.075)\end{array}$ & 0.140 \\
\hline & None & $20(14.9)$ & $6(4.5)$ & $\begin{array}{l}3.948 \\
(1.528, \\
10.201)\end{array}$ & $0.005^{*}$ \\
\hline
\end{tabular}

"Variables which show significant association during the bivariate analysis at $\mathrm{P}<$ 0.25 .

Table 3: Univariate analysis for the association between water supply and hygiene related factors and diarrheal diseases, Jimma town ART clinics, March 2013.

\begin{tabular}{|c|c|c|c|c|c|}
\hline Variable & Categories & $\begin{array}{l}\text { Cases } \\
\text { No (\%) }\end{array}$ & $\begin{array}{l}\text { Controls } \\
\text { No (\%) }\end{array}$ & $\begin{array}{l}\text { COR } \\
(95 \% \mathrm{Cl})\end{array}$ & P-value \\
\hline \multicolumn{6}{|c|}{ Household latrine } \\
\hline & Yes & $\begin{array}{l}120 \\
(89.6)\end{array}$ & $119(88.8)$ & 1.000 & \\
\hline & No & $14(10.4)$ & $15(11.2)$ & $\begin{array}{l}1.080 \\
(0.500 \\
2.336)\end{array}$ & 0.844 \\
\hline \multicolumn{6}{|c|}{ Type of latrine } \\
\hline & Flush /WC & $8(6.7)$ & $3(2.5)$ & 1.000 & \\
\hline & Pit Latrine & $\begin{array}{l}105 \\
(87.5)\end{array}$ & $102(85.7)$ & $\begin{array}{l}0.386 \\
(0.100 \\
1.496)\end{array}$ & 0.168 \\
\hline & VIP Latrine & $7(5.8)$ & $14(11.8)$ & $\begin{array}{l}0.188 \\
(0.038,0.93 \\
6)\end{array}$ & $0.041^{*}$ \\
\hline \multicolumn{6}{|c|}{ Latrine cover presence } \\
\hline & Present & $34(25.6)$ & $52(38.8)$ & 1.000 & \\
\hline & Absent & $99(74.4)$ & $82(61.2)$ & $\begin{array}{l}1.794 \\
(1.068 \\
3.014)\end{array}$ & $0.027^{*}$ \\
\hline \multicolumn{6}{|c|}{ Hand washing facility near latrine } \\
\hline & Present & $21(15.8)$ & $16(11.9)$ & 1.000 & \\
\hline & Absent & $\begin{array}{l}112 \\
(84.2)\end{array}$ & $118(88.1)$ & $\begin{array}{l}0.723 \\
(0.359 \\
1.456)\end{array}$ & 0.364 \\
\hline \multicolumn{6}{|c|}{ Solid waste disposal site } \\
\hline & On field & $37(27.6)$ & 40 (29.9) & 1.000 & \\
\hline
\end{tabular}

\begin{tabular}{|l|l|l|l|l|l|}
\hline & Pit & $46(34.3)$ & $54(40.3)$ & $\begin{array}{l}0.921 \\
(0.508, \\
1.670)\end{array}$ & 0.786 \\
\hline & $\begin{array}{l}\text { Garbage } \\
\text { container }\end{array}$ & $26(19.4)$ & $7(5.2)$ & $\begin{array}{l}4.015 \\
(1.558, \\
10.349)\end{array}$ & $0.004^{*}$ \\
\hline & Burn & $25(18.7)$ & $33(24.6)$ & $\begin{array}{l}0.819 \\
(0.413, \\
1.626)\end{array}$ & 0.568 \\
& & & & \\
\hline
\end{tabular}

Pets in the house

\begin{tabular}{|l|l|l|l|l|l|}
\hline & Present & $59(44.0)$ & $46(34.3)$ & $\begin{array}{l}1.505 \\
(0.919, \\
2.465)\end{array}$ & $0.104^{*}$ \\
\hline & Absent & $75(56.0)$ & $88(65.7)$ & 1.000 & \\
\hline
\end{tabular}

Contact with pets

\begin{tabular}{|l|l|l|l|l|l|}
\hline & Present & $58(98.3)$ & $43(93.5)$ & $\begin{array}{l}4.047 \\
(0.407, \\
40.251)\end{array}$ & $0.233^{*}$ \\
\hline & Absent & $1(1.7)$ & $3(6.5)$ & 1.000 & \\
\hline
\end{tabular}

Insects and rats at home

\begin{tabular}{|l|l|l|l|l|l|}
\hline & Present & $\begin{array}{l}128 \\
(95.5)\end{array}$ & $120(89.6)$ & $\begin{array}{l}2.489 \\
(0.926, \\
6.687)\end{array}$ & $0.071^{*}$ \\
\hline & Absent & $6(4.5)$ & $14(10.4)$ & 1.000 & \\
\hline
\end{tabular}

Refrigerator in the house

\begin{tabular}{|l|l|l|l|l|l|}
\hline & Present & $20(14.9)$ & $39(29.1)$ & 1.000 & \\
\hline & Absent & $\begin{array}{l}114 \\
(85.1)\end{array}$ & $95(70.9)$ & $\begin{array}{l}2.340 \\
(1.279, \\
4.280)\end{array}$ & $0.006^{*}$ \\
& & & & & \\
\hline
\end{tabular}

Frequency of cooking

\begin{tabular}{|l|l|l|l|l|l|}
\hline & $1-2$ & $\begin{array}{l}110 \\
(82.1)\end{array}$ & $109(81.3)$ & $\begin{array}{l}1.051 \\
(0.566, \\
1.953)\end{array}$ & 0.874 \\
\hline & $>3$ & $24(17.9)$ & $25(18.7)$ & 1.000 & \\
\hline
\end{tabular}

Eat Raw/uncooked food

\begin{tabular}{|l|l|l|l|l|l|}
\hline & Yes & $34(25.4)$ & $18(13.4)$ & $\begin{array}{l}2.191 \\
(1.166, \\
4.117)\end{array}$ & $0.015^{*}$ \\
\hline & No & $\begin{array}{l}100 \\
(74.6)\end{array}$ & $116(86.6)$ & 1.000 & \\
\hline
\end{tabular}

"Variables which show significant association during the bivariate analysis at $\mathrm{P}<0.25$.

Table 4: Univariate analysis for the association between Sanitation related factors and diarrheal diseases, Jimma town ART clinics, March 2013.

\section{Clinical factors}

Higher proportion of cases had previous hospitalization history $(\mathrm{P}=0.002)$, previous GIT disorder $(\mathrm{P}<0.001)$, other opportunistic infections $(\mathrm{P}<0.001)$, history of diarrhea $(\mathrm{P}<0.001)$, history of poor 
Citation: Ewnetu H, Kassahun W, Hiko D (2014) Determinants of Diarrheal Disease among Adult People Living with HIVIAIDS Attending ART Clinics in Jimma Town, South-Western Ethiopia: A Case Control Study. J AIDS Clin Res 5: 380. doi:10.4172/2155-6113.1000380

Page 6 of 9

adherence $(\mathrm{P}=0.093)$, and not on cotrimoxazole prophylaxis $(\mathrm{P}=0.144)$ compared to controls. However, there was no significant difference between cases and controls concerning CD4 count, WHO stage and being on ART (Table 5).

\section{Variables that have shown significant association at bivariate analysis}

During bivariate analysis, age, sex, occupation, educational status, history of khat chewing in the last one month, no of usual members of the household, source of energy for cooking, water source, point and distance from the house, type of water storage container, treatment of non-potable water, distance of water source from the latrine, habit of hand washing before food preparation, facility used for hand washing, hand washing after defecation, type of latrine, solid waste disposal site, non-use of latrine cover, presence of pets in the house, contact with pets, presence of insects and rats at home, absence of refrigerator in the house, eating raw/uncooked food, previous history of hospitalization, previous GIT disorder, other opportunistic infection, history of diarrhea, not on cotrimoxazole prophylaxis and history of poor adherence were significantly associated with diarrheal disease (Tables 1-5).

\begin{tabular}{|c|c|c|c|c|c|}
\hline Variable & Categories & $\begin{array}{l}\text { Cases } \\
\text { No (\%) }\end{array}$ & $\begin{array}{l}\text { Controls } \\
\text { No (\%) }\end{array}$ & $\begin{array}{l}\text { COR } \\
(95 \% \mathrm{Cl})\end{array}$ & P-value \\
\hline \multicolumn{6}{|c|}{ CD4 count } \\
\hline & $<200$ & $11(8.8)$ & $13(10.7)$ & $\begin{array}{l}0.802 \\
(0.344 \\
1.866)\end{array}$ & 0.608 \\
\hline & $>200$ & $114(91.2)$ & $108(89.3)$ & 1.000 & \\
\hline \multicolumn{6}{|c|}{ WHO stage } \\
\hline & Stage $1 / I I$ & $100(74.6)$ & $125(93.3)$ & 1.000 & \\
\hline & Stage III/IV & $34(25.4)$ & $9(6.7)$ & $\begin{array}{l}1.352 \\
(0.629 \\
2.908)\end{array}$ & 0.440 \\
\hline \multicolumn{6}{|c|}{ Previous Hospitalization history } \\
\hline & Yes & $20(14.9)$ & $4(3.0)$ & $\begin{array}{l}5.702 \\
(1.893 \\
17.173)\end{array}$ & $0.002^{*}$ \\
\hline & No & $114(85.1)$ & $130(97.0)$ & 1.000 & \\
\hline \multicolumn{6}{|c|}{ Previous GIT Disorder } \\
\hline & Yes & $51(38.1)$ & $12(9.0)$ & $\begin{array}{l}6.24 \\
(3.140 \\
12.429)\end{array}$ & $<0.001^{*}$ \\
\hline & No & $83(61.9)$ & $122(91.0)$ & 1.000 & \\
\hline \multicolumn{6}{|c|}{ Other Opportunistic Infection } \\
\hline & Yes & $91(67.9)$ & $42(31.3)$ & $\begin{array}{l}4.636 \\
(2.771 \\
7.755)\end{array}$ & $<0.001^{*}$ \\
\hline & No & $43(32.1)$ & $92(68.7)$ & 1.000 & \\
\hline \multicolumn{6}{|c|}{ History of Diarrhea } \\
\hline
\end{tabular}

\begin{tabular}{|c|c|c|c|c|c|}
\hline & Yes & $80(59.7)$ & $29(21.6)$ & $\begin{array}{l}5.364 \\
(3.136, \\
9.176)\end{array}$ & $<0.001^{*}$ \\
\hline & No & $54(40.3)$ & $105(78.4)$ & 1.000 & \\
\hline \multicolumn{6}{|c|}{ Currently taking ART } \\
\hline & Yes & $106(79.1)$ & $105(78.4)$ & $\begin{array}{l}1.046 \\
(0.582 \\
1.877)\end{array}$ & 0.881 \\
\hline & No & $28(20.9)$ & $29(21.6)$ & 1.000 & \\
\hline \multicolumn{6}{|c|}{ Currently taking Cotrimoxazole Prophylaxis } \\
\hline & Yes & $88(65.7)$ & 99 (73.9) & 1.000 & \\
\hline & No & $46(34.3)$ & $35(26.1)$ & $\begin{array}{l}1.479 \\
(0.875 \\
2.500)\end{array}$ & $0.144^{*}$ \\
\hline \multicolumn{6}{|c|}{ History of Poor Adherence } \\
\hline & Yes & $8(7.6)$ & $16(15.1)$ & 1.000 & \\
\hline & No & $97(92.4)$ & $90(84.9)$ & $\begin{array}{l}2.156 \\
(0.880 \\
5.280)\end{array}$ & $0.093^{*}$ \\
\hline $\begin{array}{l}\text { *Variables } \\
\mathrm{P}<0.25\end{array}$ & & icant & tion $\mathrm{d}$ & $\mathrm{b}$ & analy \\
\hline
\end{tabular}

Table 5: Univariate analysis for the association between clinical factors and diarrheal diseases, Jimma town ART clinics, March 2013.

\section{Factors independently associated with diarrheal disease}

After adjustment for potential confounders, males had twice the odds of presenting with diarrhea compared $(\mathrm{AOR}=2.276$; $95 \%$; $\mathrm{CI}$ : $1.079,4.800 ; \mathrm{P}=0.031$ ) with females.

Use of public water was strongly associated with diarrhea, with fivefold increased odds of diarrhea compared with individuals who use private water point $(\mathrm{AOR}=4.972$; 95\% CI: 2.408, 10.266; $\mathrm{P}<0.001)$. The finding also implied that, non-use of latrine cover was higher among cases, associated with 2.7 times increased odds compared to controls (AOR=2.763; 95\% CI:1.276, 5.980; $\mathrm{P}=0.010$ ). Similarly, individuals who dispose solid waste inside garbage container had seven fold increased odds for occurrence of diarrhea compared with individuals who dispose on open field (AOR=7.676; 95\%; CI:1.776, 33.188). Having pets in the house had twice the odds of presenting with diarrhea compared with no pets in the house (AOR=2.260; 95\%; CI: $1.153,4.427 ; \mathrm{P}=0.018)$ and individuals who did not have refrigerator in the house had three fold increased odds of presenting with diarrhea compared with individuals who had refrigerator in the house (AOR=3.343 95\%; CI:1.274, 8.774; $\mathrm{P}=0.014)$.

History of previous GIT disorder was strongly associated with diarrhea, with four fold increased odds of diarrhea compared with individuals who had no previous GIT disorder $(\mathrm{AOR}=4.254 ; 95 \%$; $\mathrm{CI}$ : $1.647,10.987 ; \mathrm{P}=0.003$ ). In addition, previous history of diarrhea was higher among cases, associated with four times increased odds (AOR=3.966; 95\%; CI:1.896, 8.295; $\mathrm{P}<0.001$ ) compared to controls (Table 6).

Distance of water from the house, type of house hold latrine, having contact with pets/animals, ART regimen and history of poor 
Page 7 of 9

adherence were not considered in multiple logistic regressions because of having small cell counts or missing values.

\section{Discussion}

Knowledge on the possible determinant factors is important for proper management and prevention strategy of diarrheal diseases. The findings of this study will be contrasted and discussed in line with the existing body of knowledge in the literature as follows.

This study showed that men were more at risk of diarrhea than women. Probably, differences in study area and population could contribute to the difference between the current study and that of study conducted in Switzerland and South Africa [8,9]. Studies conducted in India (New Delhi and Karnataka) revealed no significant association between sex of the respondent and diarrheal disease occurrence $[1,10]$. This study's finding replicates the finding of study done in New York hospitals which suggested that men are more likely to be admitted due to diarrheal disease compared to women [11]. This might be because men can easily be exposed to contaminated, undercooked/raw food and water while feeding outside home which is found to be the risk factor of diarrheal disease in study done in Karnataka, India but did not show significant association in multivariate analysis of current study. In addition, men may have limited access to water and sanitation facilities in their work area in which case simple sanitary measures like hand washing may not be practiced.

\begin{tabular}{|c|c|c|c|c|c|}
\hline Variable & Categories & $\begin{array}{l}\text { Cases } \\
\text { No (\%) }\end{array}$ & $\begin{array}{l}\text { Controls } \\
\text { No (\%) }\end{array}$ & $\begin{array}{l}\text { COR } \\
(95 \% \mathrm{CI})\end{array}$ & $\begin{array}{l}\text { AOR } \\
(95 \% \mathrm{CI})\end{array}$ \\
\hline \multicolumn{6}{|l|}{ Sex } \\
\hline & Male & $44(32.8)$ & $31(23.1)$ & $\begin{array}{l}1.624 \\
(0.94, \\
2.786)\end{array}$ & $\begin{array}{l}2.276 \\
(1.079 \\
4.800)^{*}\end{array}$ \\
\hline & Female & $90(67.2)$ & $103(76.9)$ & 1.000 & \\
\hline \multicolumn{6}{|c|}{ Water point } \\
\hline & Private & $46(34.3)$ & $82(61.2)$ & 1.000 & \\
\hline & Public & $88(65.7)$ & $52(38.8)$ & $\begin{array}{l}3.017 \\
(1.834, \\
4.963)\end{array}$ & $\begin{array}{l}4.972 \\
(2.408 \\
10.266)^{*}\end{array}$ \\
\hline \multicolumn{6}{|c|}{ Latrine cover } \\
\hline & Yes & $34(25.6)$ & $52(38.8)$ & 1.000 & \\
\hline & No & $99(74.4)$ & $82(61.2)$ & $\begin{array}{l}1.794 \\
(1.068 \\
3.014)\end{array}$ & $\begin{array}{l}2.763 \\
(1.276,5.98 \\
0)^{*}\end{array}$ \\
\hline \multicolumn{6}{|c|}{ Solid waste disposal site } \\
\hline & On field & $37(27.6)$ & $40(29.9)$ & 1.000 & \\
\hline & Pit & $46(34.3)$ & $54(40.3)$ & $\begin{array}{l}0.921 \\
(0.508 \\
1.670)\end{array}$ & $\begin{array}{l}1.168 \\
(0.539,2.53 \\
1)\end{array}$ \\
\hline & $\begin{array}{l}\text { Garbage } \\
\text { container }\end{array}$ & $26(19.4)$ & $7(5.2)$ & $\begin{array}{l}4.015 \\
(1.558 \\
10.349)\end{array}$ & $\begin{array}{l}7.676 \\
(1.776, \\
33.188)^{*}\end{array}$ \\
\hline
\end{tabular}

\begin{tabular}{|c|c|c|c|c|c|}
\hline & Burn & $25(18.7)$ & $33(24.6)$ & \begin{tabular}{|l}
0.819 \\
$(0.413$ \\
$1.626)$
\end{tabular} & $\begin{array}{l}0.290 \\
(0.108 \\
0.780)^{*}\end{array}$ \\
\hline \multicolumn{6}{|c|}{ Pets in the house } \\
\hline & Present & $59(44.0)$ & $46(34.3)$ & $\begin{array}{l}1.505 \\
(0.919 \\
2.465)\end{array}$ & $\begin{array}{l}2.260 \\
(1.153 \\
4.427)^{*}\end{array}$ \\
\hline & Absent & $75(56.0)$ & $88(65.7)$ & 1.000 & \\
\hline \multicolumn{6}{|c|}{ Refrigerator } \\
\hline & Present & $20(14.9)$ & $39(29.1)$ & 1.000 & \\
\hline & Absent & $114(85.1)$ & $95(70.9)$ & $\begin{array}{l}2.340 \\
(1.279, \\
4.280)\end{array}$ & $\begin{array}{l}3.343 \\
(1.274, \\
8.774)^{*}\end{array}$ \\
\hline \multicolumn{6}{|c|}{ Previous GIT disorder } \\
\hline & Yes & $51(38.1)$ & $12(9.0)$ & \begin{tabular}{|l}
6.24 \\
$(3.140$ \\
$12.429)$
\end{tabular} & $\begin{array}{l}4.254 \\
(1.647 \\
10.987)^{*}\end{array}$ \\
\hline & No & $83(61.9)$ & $122(91.0)$ & 1.000 & \\
\hline \multicolumn{6}{|c|}{ History of diarrhea } \\
\hline & Yes & $80(59.7)$ & $29(21.6)$ & \begin{tabular}{|l}
5.364 \\
$(3.136$, \\
$9.176)$
\end{tabular} & $\begin{array}{l}3.966 \\
(1.896, \\
8.295)^{*}\end{array}$ \\
\hline & No & $54(40.3)$ & $105(78.4)$ & 1.000 & \\
\hline
\end{tabular}

Table 6: Factors independently associated with diarrheal diseases, Jimma town ART clinics, March 2013.

Use of public water was directly related with diarrheal disease in this study. Associations had been previously reported between water access and diarrheal diseases $[1,8,12]$. This could be due to PLWHAs need for water access and clean water increase to protect themselves from infection or cope with the symptoms but when the water source is not private, households economize on water and tend not to wash their hands with soap. Even though hand washing after defecation $(\mathrm{P}<0.001)$ and before food preparation $(\mathrm{P}=0.025)$ had shown association with diarrheal disease during bivariate analysis, it exhibited no significant association in the final model. However meta-analysis of hand washing studies conducted in developing countries concluded that hand washing can reduce the risk of diarrhea in the general population by 42 to $44 \%$ [13].

In this study, diarrheal disease was found to be associated with nonuse of latrine cover. If the latrine/squat hole is not covered, the houseflies breed and feed on human excreta, pick diarrhea causing pathogens in the process and contaminate the food and drinks of the individuals and expose them to diarrheal disease. This is in line with the finding of cross sectional study conducted in Nekemte town which highlighted latrine/squat hole cover is protective of diarrheal disease [14]. Even though safe disposal and handling of feces is the most important factor in the prevention of diarrheal disease, no statistically significant association was found between diarrheal disease status and presence of latrine in the current study. This is probably because no great differences exist with respect to the presence of latrine between 
the cases and controls ( $90 \%$ are owners) and it may also be because of the possible interventions done in different parts of the country with regard to latrine ownership especially for PLWHAs.

In addition, we found solid waste disposal inside garbage container is associated with increased risk of diarrhea. This might be because garbage container is placed near/inside the house most of the time and it will become an ideal place for breeding of flies and different insects which in turn contaminate the food and drinks of the household. This finding is in line with study done in Ghana which explained waste storage near the home is associated with the presence of houseflies in the kitchen $(\mathrm{P}<0.001)$ [15]. In addition, when waste materials are disposed inside garbage container around house, it can easily accessed by other reservoirs and vectors of microorganisms like pets, small ruminants and rats living inside home which is found to be a risk factor of diarrheal disease among PLWHAs on study done in Karnataka [1]. We also found protective effect of burning solid waste against diarrheal disease as poor handling and disposal of wastes creates breeding grounds for pathogenic organisms and the spread of infectious diseases mainly diarrhea and this finding is in line with study conducted in Nekemte town [14].

The study also suggested that, presence of pets/animals in the house is associated with diarrhea which is supported by study findings conducted in India, Uganda and South Africa $[1,10,12,16]$ which reported having exposure to pets and animals is accounted for significant diarrheagenic parasitic burden. This might be because, pets and animals can act as reservoir of diarrhea causing organisms and presence of pets/animals inside house is a proxy for possible contact with individuals as indicated in this study in which $98.3 \%$ of cases and 93.5\% of controls who had pets in house had contact with them implying possible transmission of diarrhea causing microorganisms from animals to the individuals. Though no significant association was observed in the current study, contact with pets/animals was found to be potential risk factor of diarrheal disease as reported in the abovementioned studies.

Absence of refrigerator at home is significantly associated with diarrheal diseases in contrary to similar study conducted in India which did not found association between refrigerator ownership and diarrhea [1]. This could be due to difference in socio economic status of study participants. An implication of the finding is the possibility that the individuals who had refrigerator can reserve perishable foods, such a meat, vegetables and milk (foods which are very liable to be spoiled) for much longer periods than are otherwise impossible. It could also help to protect against the contamination by pets/animals.

Among the clinical factors, previous clinically diagnosed gastro intestinal disorder was strongly and significantly associated with diarrhea. This has been noted in previous studies $[1,5]$. There can be several explanations for the association between previous GIT disorder and diarrheal disease. First, inadequate treatment of the previous GIT pathology could recur at any time and diarrhea may be one way of manifestation. On the other hand, once the individual had GIT disorder of any type in the past, the GIT mucosa will be more liable to be irritated by any factor and develop diarrheal diseases in the future easily. Additionally, the abovementioned studies reported that presence of another opportunistic infection could also facilitate the occurrence of diarrheal diseases but it is not found to be a potential risk factor in this study.

Similarly, individuals who had a previous history of diarrhea were also at an increased risk of diarrhea as previously shown elsewhere [1].
This could be due to persistent exposure to risk factors in the home environment, to hygiene-related risk factors, or a recurrence of diarrhea that persisted due to inadequate treatment and continued carriage of the pathogen.

CD4 count and WHO stages of the participants were found to be significant risk factors of diarrheal diseases among PLWHAs in studies conducted in India, Bangkok and Ethiopia [1,10,17-19] indicating that PLWHAs whose CD4 count is $<200$ cells $/ \mathrm{mm}^{3}$ and WHO stage III/IV are at increased risk of diarrheal disease which directly indicate the level of their immunocompromisation and also their susceptibility to develop different opportunistic infections mainly diarrheal diseases. But these factors did not exhibit significant association in the current study and this might be because no great difference exists with respect to $\mathrm{CD} 4$ count and WHO staging between cases and controls.

This study has the following limitations: First, a case control study can only identify associations rather than establishing cause and effect relationship. Second, recall bias might have affected the accuracy of information especially related to substance use and hygiene related practices

\section{Conclusions}

In summary, this study identified some of the factors that facilitate the occurrence of diarrheal diseases among peoples living with HIV/ AIDS which may help to design possible intervention strategies to prevent occurrence of diarrhea among these population.

We found that diarrheal disease were associated with gender, environmental and clinical factors such as public water point, non-use of latrine covers, presence of pets in the house, solid waste disposal in garbage container, absence of refrigerator, having history of clinically diagnosed GIT disorder and previous history of diarrhea. Health professionals and Health institutions should work with different stakeholders in multidisciplinary approach including access to safe water, sanitation and hygiene as essential components of basic preventive care package in strengthened way for PLWHAs while providing routine care.

\section{Acknowledgements}

We acknowledge Jimma University for financing the study. We thank all participants for devoting their time to take part in this study.

\section{References}

1. Becker ML, Cohen CR, Cheang M, Washington RG, Blanchard JF, et al. (2007) Diarrheal disease among HIV-infected adults in Karnataka, India: evaluation of risk factors and etiology. Am J Trop Med Hyg 76: 718-722.

2. http://www.unaids.org/en/media/unaids/contentassets/documents/ unaidspublication/2011/JC2216_WorldAIDSday_report_2011_en.pdf

3. Central Statistical Agency (2012) Ethiopia Demographic and Health Survey 2011 Addis Ababa, Ethiopia and Calverton, Maryland, USA.

4. http://transition.usaid.gov/our_work/global_health/aids/Countries/ africa/hiv_summary_africa.pdf

5. Treakle A (2008) Diarrhea and HIV in the US in the post-HAART era.

6. Lule JR, Mermin J, Ekwaru JP, Malamba S, Downing R, et al. (2005) Effect of home-based water chlorination and safe storage on diarrhea among persons with human immunodeficiency virus in Uganda. Am J Trop Med Hyg 73: 926-933.

7. Bushen OY, Davenport JA, Lima AB, Piscitelli SC, Uzgiris AJ, et al. (2004) Diarrhea and reduced levels of antiretroviral drugs: improvement 
Citation: Ewnetu H, Kassahun W, Hiko D (2014) Determinants of Diarrheal Disease among Adult People Living with HIVIAIDS Attending ART Clinics in Jimma Town, South-Western Ethiopia: A Case Control Study. J AIDS Clin Res 5: 380. doi:10.4172/2155-6113.1000380

Page 9 of 9

with glutamine or alanyl-glutamine in a randomized controlled trial in northeast Brazil. Clin Infect Dis 38: 1764-1770.

8. Moshabela M, MacPherson P, Ezard N, Frean E, Mashimbye L, et al. (2012) Clinical and social determinants of diarrhoeal disease in a rural HIV/AIDS clinic, South Africa: a case-control study. Int J STD AIDS 23: 346-350.

9. Weber R, Ledergerber B, Zbinden R, Altwegg M, Pfyffer GE, et al. (1999) Enteric infections and diarrhea in human immunodeficiency virusinfected persons: prospective community-based cohort study. Swiss HIV Cohort Study. Arch Intern Med 159: 1473-1480.

10. Dwivedi KK, Prasad G, Saini S, Mahajan S, Lal S, et al. (2007) Enteric opportunistic parasites among HIV infected individuals: associated risk factors and immune status. Jpn J Infect Dis 60: 76-81.

11. Anastasi JK, Capili B (2000) HIV and diarrhea in the era of HAART: 1998 New York State hospitalizations. Am J Infect Control 28: 262-266.

12. O'Keefe EA, Wood R (1996) AIDS in Africa. Scand J Gastroenterol 220: 147-152.

13. Curtis V, Cairncross S (2003) Effect of washing hands with soap on diarrhoea risk in the community: a systematic review. Lancet Infect Dis 3: 275-281.
14. Regassa G, Birke W, Deboch B, Belachew T (2008) Environmental Determinants of Diarrhea among Under-Five Children in Nekemte Town, Western Ethiopia. Ethiop J Health Science 18: 39-45.

15. Boadi KO, Kuitunen M (2005) Environmental and health impacts of household solid waste handling and disposal practices in third world cities: the case of the Accra Metropolitan Area, Ghana. J Environ Health 68: $32-36$.

16. Nasinyama GW, McEwen SA, Wilson JB, Waltner-Toews D, Gyles CL, et al. (2000) Risk factors for acute diarrhoea among inhabitants of Kampala District, Uganda. S Afr Med J 90: 891-898.

17. Wanke CA, Cohan D, Thummakul T, Jongwuitiwes S, Grayson ML, et al. (1999) Diarrheal disease in patients infected with human immunodeficiency virus in Bangkok, Thailand. Am J Trop Med Hyg 60: 871-874.

18. Attili SV, Gulati AK, Singh VP, Varma DV, Rai M, et al. (2006) Diarrhea, CD4 counts and enteric infections in a hospital - based cohort of HIVinfected patients around Varanasi, India. BMC Infect Dis 6: 39.

19. Kleinau E, Pyle FD (2012) Environmental health project. Assessing hygiene improvement. Guideline for household and community levels. 